\title{
A Survey on Wireless Body Area Networks
}

\author{
Benoît Latré - Bart Braem • Ingrid Moerman - Chris Blondia • \\ Piet Demeester
}

Received: date / Accepted: date

\begin{abstract}
The increasing use of wireless networks and the constant miniaturization of electrical devices has empowered the development of Wireless Body Area Networks (WBANs). In these networks various sensors are attached on clothing or on the body or even implanted under the skin. The wireless nature of the network and the wide variety of sensors offer numerous new, practical and innovative applications to improve health care and the Quality of Life. The sensors of a WBAN measure for example the heartbeat, the body temperature or record a prolonged electrocardiogram. Using a WBAN, the patient experiences a greater physical mobility and is no longer compelled to stay in the hospital. This paper offers a survey of the concept of Wireless Body Area Networks. First, we focus on some applications with special interest in patient monitoring. Then the communication in a WBAN and its positioning between the different technologies is discussed. An overview of the current research on the physical layer, existing MAC and network protocols is given. Further, cross layer and quality of service is discussed. As WBANs are placed on the human body and often transport private data, security is also considered. An overview of current and past projects is given. Finally, the open research issues and challenges are pointed out.
\end{abstract}

Benoît Latré, Ingrid Moerman, Piet Demeester

Department of Information Technology, Ghent University / IBBT, Gaston Crommenlaan 8 box 201, B-9050 Gent, Belgium, Tel.: +32-45-678910

Fax: $+132-45-678910$

E-mail: benoit.latre@intec.ugent.com

Bart Braem, Chris Blondia

Department of Mathematics and Computer Science, University of Antwerp / IBBT,

Middelheimlaan 1, B-2020, Antwerp, Belgium

\section{Introduction}

The aging population in many developed countries and the rising costs of health care have triggered the introduction of novel technology-driven enhancements to current health care practices. For example, recent advances in electronics have enabled the development of small and intelligent (bio-) medical sensors which can be worn on or implanted in the human body. These sensors need to send their data to an external medical server where it can be analyzed and stored. Using a wired connection for this purpose turns out to be too cumbersome and involves a high cost for deployment and maintenance. However, the use of a wireless interface enables an easier application and is more cost efficient [1]. The patient experiences a greater physical mobility and is no longer compelled to stay in a hospital. This process can be considered as the next step in enhancing the personal health care and in coping with the costs of the health care system. Where eHealth is defined as the health care practice supported by electronic processes and communication, the health care is now going a step further by becoming mobile. This is referred to as mHealth [2]. In order to fully exploit the benefits of wireless technologies in telemedicine and mHealth, a new type of wireless network emerges: a wireless on-body network or a Wireless Body Area Network (WBAN). This term was first coined by Van Dam et al. in 2001 [3] and received the interest of several researchers [4-8].

A Wireless Body Area Network consists of small, intelligent devices attached on or implanted in the body which are capable of establishing a wireless communication link. These devices provide continuous health monitoring and real-time feedback to the user or medical personnel. Furthermore, the measurements can be 
recorded over a longer period of time, improving the quality of the measured data [9].

Generally speaking, two types of devices can be distinguished: sensors and actuators. The sensors are used to measure certain parameters of the human body, either externally or internally. Examples include measuring the heartbeat, body temperature or recording a prolonged electrocardiogram (ECG). The actuators (or actors) on the other hand take some specific actions according to the data they receive from the sensors or through interaction with the user. E.g., an actuator equipped with a built-in reservoir and pump administers the correct dose of insulin to give to diabetics based on the glucose level measurements. Interaction with the user or other persons is usually handled by a personal device, e.g. a PDA or a smart phone which acts as a sink for data of the wireless devices.

In order to realize communication between these devices, techniques from Wireless Sensor Networks (WSNs) and ad hoc networks could be used. However, because of the typical properties of a WBAN, current protocols designed for these networks are not always well suited to support a WBAN. The following illustrates the differences between a Wireless Sensor Network and a Wireless Body Area Network:

- The devices used have limited energy resources available as they have a very small form factor (often less than $\left.1 \mathrm{~cm}^{3}[10]\right)$. Furthermore, for most devices it is impossible to recharge or change the batteries although a long lifetime of the device is wanted (up to several years or even decades for implanted devices). Hence, the energy resources and consequently the computational power and available memory of such devices will be limited;

- All devices are equally important and devices are only added when they are needed for an application (i.e. no redundant devices are available);

- An extremely low transmit power per node is needed to minimize interference and to cope with health concerns [11];

- The propagation of the waves takes place in or on a (very) lossy medium, the human body. As a result, the waves are attenuated considerably before they reach the receiver;

- The devices are located on the human body that can be in motion. WBANs should therefore be robust against frequent changes in the network topology;

- The data mostly consists of medical information. Hence, high reliability and low delay is required;

- Stringent security mechanisms are required in order to ensure the strictly private and confidential character of the medical data;
- And finally the devices are often very heterogeneous, may have very different demands or may require different resources of the network in terms of data rates, power consumption and reliability.

When referring to a WBAN where each node comprises a biosensor or a medical device with sensing unit, some researchers use the name Body Area Sensor Network (BASN) or in short Body Sensor Network (BSN) instead of WBAN [12]. These networks are very similar to each other and share the same challenges and properties. In the following, we will use the term WBAN which is also the one used by the IEEE [13].

In this article we present a survey of the state of the art in Wireless Body Area Networks. Our aim is to provide a better understanding of the current research issues in this emerging field. The remainder of this paper is organized as follows. First, the patient monitoring application is discussed in Section 2. Next, the characteristics of the communication and the positioning of WBANs amongst other wireless technologies is discussed in Section 4. Section 5 gives an overview of the properties of the physical layer and the issues of communicating near or in the body. Existing protocols for the MAC-layer and network layer are discussed in Section 6 and Section 7 respectively. Section 8 deals with cross-layer protocols available for WBANs. The Quality of Service and possible security mechanisms are treated in Section 9 and 10. An overview of existing projects is given in Section 11. Finally, the open research issues are discussed in Section 12 and Section 13 concludes the paper.

\section{Patient Monitoring}

The main cause of death in the world is CardioVascular Disease (CVD), representing $30 \%$ of all global deaths. According to the World Health Organization, worldwide about 17.5 million people die of heart attacks or strokes each year; in 2015, almost 20 million people will die from CVD. These deaths can often be prevented with proper health care [14]. Worldwide, more than 246 million people suffer from diabetes, a number that is expected to rise to 380 million by 2025 [15]. Frequent monitoring enables proper dosing and reduces the risk of fainting and in later life blindness, loss of circulation and other complications [15].

These two examples already illustrate the need for continuous monitoring and the usefulness of WBANs. Numerous other examples of diseases would benefit from continuous or prolonged monitoring, such as hypertension, asthma, Alzheimer's disease, Parkinson's disease, renal failure, post-operative monitoring, stress-monitoring, 
prevention of sudden infant death syndrome etc $[9,16$, 17]. These applications can be considered as an indicator for the size of the market for WBANs. The number of people suffering from diabetics or CVD and the percentage of people in the population age 60 years and older will grow in the future. Even without any further increase in world population by 2025 this would mean a very large number of potential customers. WBAN technology could provide the connectivity to support the elderly in managing their daily life and medical conditions [18]. A WBAN allows continuous monitoring of the physiological parameters. Whether the patient is in the hospital, at home or on the move, the patient will no longer need to stay in bed, but will be able to move around freely. Furthermore, the data obtained during a large time interval in the patient's natural environment offers a clearer view to the doctors than data obtained during short stays at the hospital [9].

An example of a medical WBAN used for patient monitoring is shown in Figure 1. Several sensors are placed in clothes, directly on the body or under the skin of a person and measure the temperature, blood pressure, heart rate, ECG, EEG, respiration rate, $\mathrm{SpO}_{2}-$ levels etc. Next to sensing devices, the patient has actuators which act as drug delivery systems. The medicine can be delivered on predetermined moments, triggered by an external source (i.e. a doctor who analyzes the data) or immediately when a sensor notices a problem. One example is the monitoring of the glucose level in the blood of diabetics. If the sensor monitors a sudden drop of glucose, a signal can be sent to the actuator in order to start the injection of insulin. Consequently, the patient will experience fewer nuisances from his disease. Another example of an actuator is a spinal cord stimulator implanted in the body for long-term pain relief [19].

A WBAN can also be used to offer assistance to the disabled. For example, a paraplegic can be equipped with sensors determining the position of the legs or with sensors attached to the nerves [20]. In addition, actuators positioned on the legs can stimulate the muscles. Interaction between the data from the sensors and the actuators makes it possible to restore the ability to move. Another example is aid for the visually impaired. An artificial retina, consisting of a matrix of micro sensors, can be implanted into the eye beneath the surface of the retina. The artificial retina translates the electrical impulses into neurological signals. The input can be obtained locally from light sensitive sensors or by an external camera mounted on a pair of glasses [21].

Another area of application can be found in the domain of public safety where the WBAN can be used by firefighters, policemen or in a military environment [22].

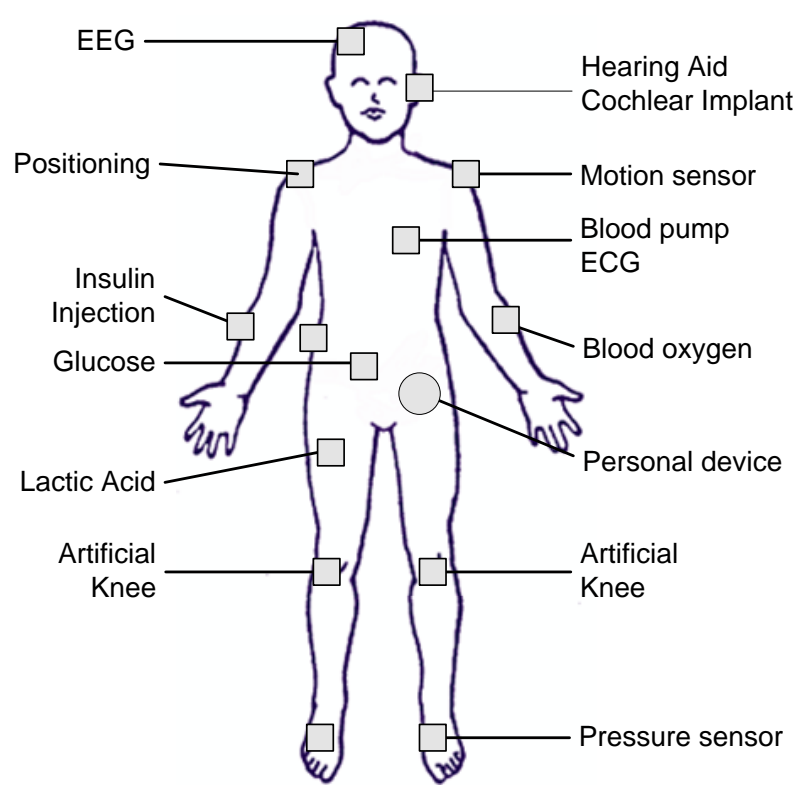

Fig. 1 Example of patient monitoring in a Wireless Body Area Network.

The WBAN monitors for example the level of toxics in the air and warns the firefighters or soldiers if a life threatening level is detected. The introduction of a WBAN further enables to tune more effectively the training schedules of professional athletes.

Next to purely medical applications, a WBAN can include appliances such as an MP3-player, head-mounted (computer) displays, a microphone, a camera, advanced human-computer interfaces such as a neural interface etc [20]. As such, the WBAN can also be used for gaming purposes and in virtual reality.

This small overview already shows the myriad of possibilities where WBANs are useful. The main characteristic of all these applications is that WBANs improve the user's Quality of Life.

\section{Taxonomy and Requirements}

The applications described in the previous section indicate that a WBAN consists of several heterogeneous devices. In this section an overview of the different types of devices used in a WBAN will be given. Further the requirements and challenges are discussed. These include the wide variability of data rates, the restricted energy consumption, the need for quality of service and reliability, ease-of-use by medical professionals and security and privacy issues. 
3.1 Types of Devices

(Wireless) Sensor node:

A device that responds to and gathers data on physical stimuli, processes the data if necessary and reports this information wirelessly. It consists of several components: sensor hardware, a power unit, a processor, memory and a transmitter or transceiver [23].

(Wireless) Actuator node:

A device that acts according to data received from the sensors or through interaction with the user. The components of an actuator are similar to the sensor's: actuator hardware (e.g. hardware for medicine administration, including a reservoir to hold the medicine), a power unit, a processor, memory and a receiver or transceiver.

(Wireless) Personal Device (PD):

A device that gathers all the information acquired by the sensors and actuators and informs the user (i.e. the patient, a nurse, a GP etc.) via an external gateway, an actuator or a display/LEDS on the device. The components are a power unit, a (large) processor, memory and a transceiver. This device is also called a Body Control Unit (BCU) [4], bodygateway or a sink. In some implementations, a Personal Digital Assistant (PDA) or smart phone is used.

Many different types of sensors and actuators are used in a WBAN. The main use of all these devices is to be found in the area of health applications. In the following, the term nodes refers to both the sensor as actuator nodes.

The number of nodes in a WBAN is limited by nature of the network. It is expected that the number of nodes will be in the range of 20-50 [6,24].

\subsection{Data Rates}

Due to the strong heterogeneity of the applications, data rates will vary strongly, ranging from simple data at a few kbit/s to video streams of several Mbit/s. Data can also be sent in bursts, which means that it is sent at higher rate during the bursts.

The data rates for the different applications are given in in Table 1 and are calculated by means of the sampling rate, the range and the desired accuracy of the measurements $[25,26]$. Overall, it can be seen that the application data rates are not high. However, if one has a WBAN with several of these devices (i.e. a dozen motion sensors, ECG, EMG, glucose monitoring etc.) the aggregated data rate easily reaches a few Mbps, which
Table 1 Examples of medical WBAN applications [21,25-27]

\begin{tabular}{|l|ccc|}
\hline Application & Data Rate & Bandwidth & Accuracy \\
\hline \hline ECG (12 leads) & $288 \mathrm{kbps}$ & $100-1000 \mathrm{~Hz}$ & $12 \mathrm{bits}$ \\
ECG (6 leads) & $71 \mathrm{kbps}$ & $100-500 \mathrm{~Hz}$ & $12 \mathrm{bits}$ \\
EMG & $320 \mathrm{kbps}$ & $0-10,000 \mathrm{~Hz}$ & $16 \mathrm{bits}$ \\
EEG (12 leads) & $43.2 \mathrm{kbps}$ & $0-150 \mathrm{~Hz}$ & $12 \mathrm{bits}$ \\
Blood saturation & $16 \mathrm{bps}$ & $0-1 \mathrm{~Hz}$ & $8 \mathrm{bits}$ \\
Glucose monitoring & $1600 \mathrm{bps}$ & $0-50 \mathrm{~Hz}$ & $16 \mathrm{bits}$ \\
Temperature & $120 \mathrm{bps}$ & $0-1 \mathrm{~Hz}$ & $8 \mathrm{bits}$ \\
Motion sensor & $35 \mathrm{kbps}$ & $0-500 \mathrm{~Hz}$ & $12 \mathrm{bits}$ \\
Cochlear implant & $100 \mathrm{kbps}$ & - & - \\
Artificial retina & $50-700 \mathrm{kbps}$ & - & - \\
Audio & $1 \mathrm{Mbps}$ & - & - \\
Voice & $50-100 \mathrm{kbps}$ & - & - \\
\hline
\end{tabular}

is a higher than the raw bit rate of most existing low power radios.

The reliability of the data transmission is provided in terms of the necessary bit error rate (BER) which is used as a measure for the number of lost packets. For a medical device, the reliability depends on the data rate. Low data rate devices can cope with a high BER (e.g. $\left.10^{-4}\right)$, while devices with a higher data rate require a lower BER (e.g. $10^{-10}$ ). The required BER is also dependent on the criticalness of the data.

\subsection{Energy}

Energy consumption can be divided into three domains: sensing, (wireless) communication and data processing [23]. The wireless communication is likely to be the most power consuming. The power available in the nodes is often restricted. The size of the battery used to store the needed energy is in most cases the largest contributor to the sensor device in terms of both dimensions and weight. Batteries are, as a consequence, kept small and energy consumption of the devices needs to be reduced. In some applications, a WBAN's sensor/actuator node should operate while supporting a battery life time of months or even years without intervention. For example, a pacemaker or a glucose monitor would require a lifetime lasting more than 5 years. Especially for implanted devices, the lifetime is crucial. The need for replacement or recharging induces a cost and convenience penalty which is undesirable not only for implanted devices, but also for larger ones.

The lifetime of a node for a given battery capacity can be enhanced by scavenging energy during the operation of the system. If the scavenged energy is larger 
than the average consumed energy, such systems could run eternally. However, energy scavenging will only deliver small amounts of energy $[5,28]$. A combination of lower energy consumption and energy scavenging is the optimal solution for achieving autonomous Wireless Body Area Networks. For a WBAN, energy scavenging from on-body sources such as body heat and body vibration seems very well suited. In the former, a thermoelectric generator (TEG) is used to transform the temperature difference between the environment and the human body into electrical energy [27]. The latter uses for example the human gait as energy source [29].

During communication the devices produce heat which is absorbed by the surrounding tissue and increases the temperature of the body. In order to limit this temperature rise and in addition to save the battery resources, the energy consumption should be restricted to a minimum. The amount of power absorbed by the tissue is expressed by the specific absorption rate (SAR). Since the device may be in close proximity to, or inside, a human body, the localized SAR could be quite large. The localized SAR into the body must be minimized and needs to comply with international and local SAR regulations. The regulation for transmitting near the human body is similar to the one for mobile phones, with strict transmit power requirements $[11,30]$

\subsection{Quality of Service and Reliability}

Proper quality of service (QoS) handling is an important part in the framework of risk management of medical applications. A crucial issue is the reliability of the transmission in order to guarantee that the monitored data is received correctly by the health care professionals. The reliability can be considered either end-to-end or on a per link base. Examples of reliability include the guaranteed delivery of data (i.e. packet delivery ratio), in-order-delivery, ... Moreover, messages should be delivered in reasonable time. The reliability of the network directly affects the quality of patient monitoring and in a worst case scenario it can be fatal when a life threatening event has gone undetected [31].

\subsection{Usability}

In most cases, a WBAN will be set up in a hospital by medical staff, not by ICT-engineers. Consequently, the network should be capable of configuring and maintaining itself automatically, i.e. self-organization an selfmaintenance should be supported. Whenever a node is put on the body and turned on, it should be able to join the network and set up routes without any external intervention. The self-organizing aspect also includes the problem of addressing the nodes. An address can be configured at manufacturing time (e.g. the MACaddress) or at setup time by the network itself. Further, the network should be quickly reconfigurable, for adding new services. When a route fails, a back up path should be set up.

The devices may be scattered over and in the whole body. The exact location of a device will depend on the application, e.g. a heart sensor obviously must be placed in the neighborhood of the heart, a temperature sensor can be placed almost anywhere. Researchers seem to disagree on the ideal body location for some sensor nodes, i.e. motion sensors, as the interpretation of the measured data is not always the same [32]. The network should not be regarded as a static one. The body may be in motion (e.g. walking, running, twisting etc.) which induces channel fading and shadowing effects.

The nodes should have a small form factor consistent with wearable and implanted applications. This will make WBANs invisible and unobtrusive.

\subsection{Security and Privacy}

The communication of health related information between sensors in a WBAN and over the Internet to servers is strictly private and confidential [33] and should be encrypted to protect the patient's privacy. The medical staff collecting the data needs to be confident that the data is not tampered with and indeed originates from that patient. Further, it can not be expected that an average person or the medical staff is capable of setting up and managing authentication and authorization processes. Moreover the network should be accessible when the user is not capable of giving the password (e.g. to guarantee accessibility by paramedics in trauma situations). Security and privacy protection mechanisms use a significant part of the available energy and should therefor be energy efficient and lightweight.

\section{Positioning WBANs}

The development and research in the domain of WBANs is only at an early stage. As a consequence, the terminology is not always clearly defined. In literature, protocols developed for WBANs can span from communication between the sensors on the body to communication from a body node to a data center connected to the Internet. In order to have clear understanding, we propose the following definitions: intra-body communication and extra-body communication. An example is 


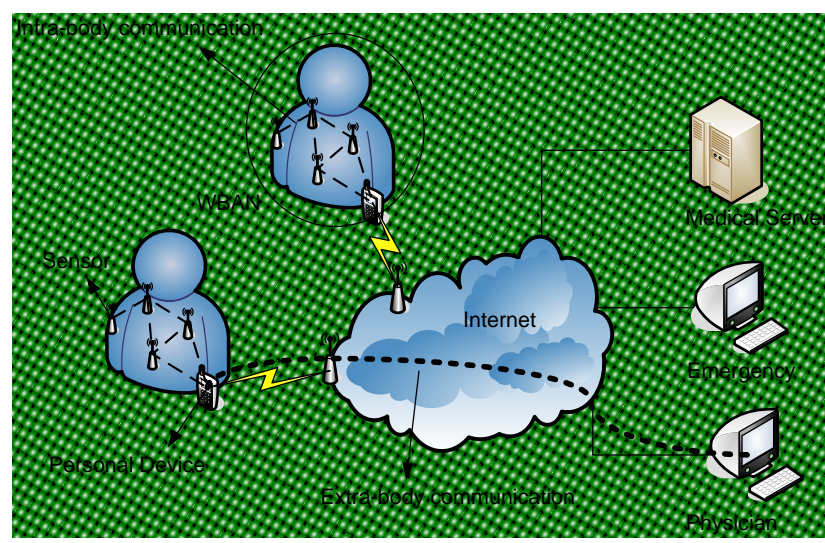

Fig. 2 Example of intra-body and extra-body communication in a WBAN.

shown on Figure 2. The former controls the information handling on the body between the sensors or actuators and the personal device [34-37], the latter ensures communication between the personal device and an external network [32,38-40]. Doing so, the medical data from the patient at home can be consulted by a physician or stored in a medical database. This segmentation is similar to the one defined in [40] where a multi-tiered telemedicine system is presented. Tier 1 encompasses the intra-body communication, tier 2 the extra-body communication between the personal device and the Internet and tier 3 represents the extra-body communication from the Internet to the medical server. The combination of intra-body and extra-body communication can be seen as an enabler for ubiquitous health care service provisioning. An example can be found in [41] where Utility Grid Computing is combined with a WBAN. Doing so, the data extracted from the WBAN is sent to the grid that provides access to appropriate computational services with high bandwidth and to a large collection of distributed time-varying resources.

To date, development has been mainly focused on building the system architecture and service platform for extra-body communication. Much of these implementations focus on the repackaging of traditional sensors (e.g. ECG, heart rate) with existing wireless devices. They consider a very limited WBAN consisting of only a few sensors that are directly and wirelessly connected to a personal device. Further they use transceivers with a large form factor and large antennas that are not adapted for use on a body.

In Figure 3, a WBAN is compared with other types of wireless networks, such as Wireless Personal (WPAN), Wireless Local (WLAN), Wireless Metropolitan (WMAN) and Wide Area Networks (WAN) [42]. A WBAN is operated close to the human body and its communication range will be restricted to a few meters, with typical

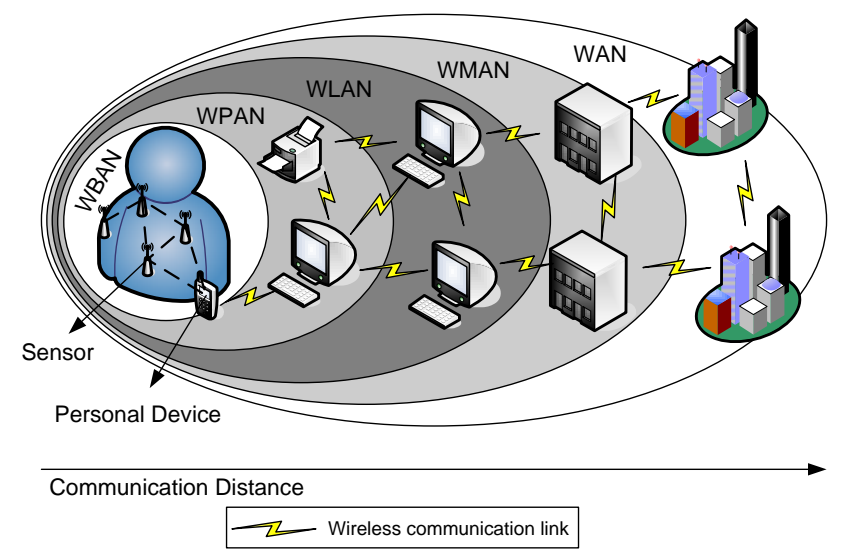

Fig. 3 Positioning of a Wireless Body Area Network in the realm of wireless networks.

values around 1-2 meters. While a WBAN is devoted to interconnection of one person's wearable devices, a WPAN is a network in the environment around the person. The communication range can reach up to 10 meters for high data rate applications and up to several dozens of meters for low data rate applications. A WLAN has a typical communication range up to hundreds of meters. Each type of network has its typical enabling technology, defined by the IEEE. A WPAN uses IEEE 802.15.1 (Bluetooth) or IEEE 802.15.4 (ZigBee), a WLAN uses IEEE 802.11 (WiFi) and a WMAN IEEE 802.16 (WiMax). The communication in a WAN can be established via satellite links.

In several papers, Wireless Body Area Networks are considered as a special type of a Wireless Sensor Network or a Wireless Sensor and Actuator Network (WSAN) with its own requirements ${ }^{1}$. However, traditional sensor networks do not tackle the specific challenges associated with human body monitoring. The human body consists of a complicated internal environment that responds to and interacts with its external surroundings, but is in a way separate and selfcontained. The human body environment not only has a smaller scale, but also requires a different type and frequency of monitoring, with different challenges than those faced by WSNs. The monitoring of medical data results in an increased demand for reliability. The ease of use of sensors placed on the body leads to a small form factor that includes the battery and antenna part, resulting in a higher need for energy efficiency. Sensor nodes can move with regard to each other, for example a sensor node placed on the wrist moves in relation to a sensor node attached to the hip. This requires mobility support. In brief, although challenges faced by WBANs

1 In the following, we will not make a distinction between a WSAN and a WSN although they have significant differences [43]. 


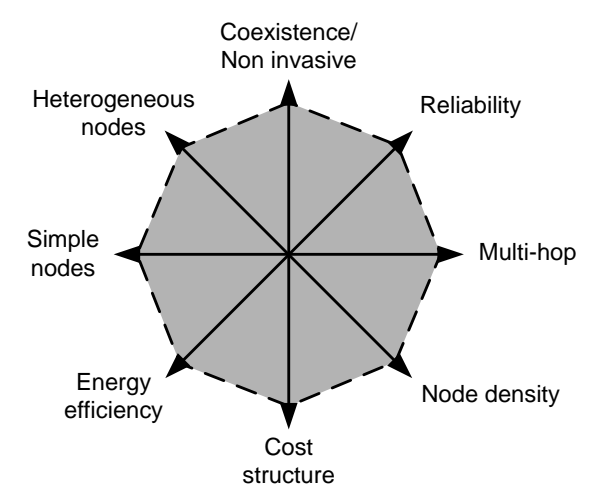

WBAN
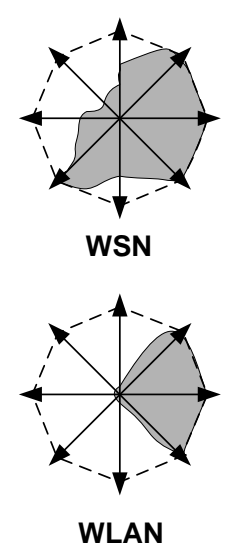

Fig. 4 Characteristics of a Wireless Body Area Network compared with Wireless Sensor Networks (WSN) and Wireless Local Area Network (WLAN). Based on [44].

are in many ways similar to WSNs, there are intrinsic differences between the two, requiring special attention. An overview of some of these differences is given in Table 2. A schematic overview of the challenges in a WBAN and a comparison with WSNs and WLANs is given in Figure 4.

\section{Physical layer}

The characteristics of the physical layer are different for a WBAN compared to a regular sensor network or an ad-hoc network due to the proximity of the human body. Tests with TelosB motes (using the CC2420 transceiver) showed lack of communications between nodes located on the chest and nodes located on the back of the patient [46]. This was accentuated when the transmit power was set to a minimum for energy savings reasons. Similar conclusions where drawn with a CC2420 transceiver in [47]: when a person was sitting on a sofa, no communication was possible between the chest and the ankle. Better results were obtained when the antenna was placed $1 \mathrm{~cm}$ above the body. As the devices get smaller and more ubiquitous, a direct connection to the personal device will no longer be possible and more complex network topologies will be needed. In this section, we will discuss the characteristics of the propagation of radio waves in a WBAN and other types of communication.

\subsection{RF communication}

Several researchers have been investigating the path loss along and inside the human body either using narrowband radio signals or Ultra Wide Band (UWB). All of them came to the conclusion that the radio signals experience great losses. Generally in wireless networks, it is known that the transmitted power drops off with $d^{\eta}$ where $d$ represents the distance between the sender and the receiver and $\eta$ the coefficient of the path loss (aka propagation coefficient) [48]. In free space, $\eta$ has a value of 2 . Other kinds of losses include fading of signals due to multi-path propagation. The propagation can be classified according to where it takes place: inside the body or along the body.

\subsubsection{In the Body}

The propagation of electromagnetic (EM) waves in the human body has been investigated in $[49,50]$. The body acts as a communication channel where losses are mainly due to absorption of power in the tissue, which is dissipated as heat. As the tissue is lossy and mostly consists of water, the EM-waves are attenuated considerably before they reach the receiver. In order to determine the amount of power lost due to heat dissipation, a standard measure of how much power is absorbed in tissue is used: the specific absorption rate (SAR). It is concluded that the path loss is very high and that, compared to the free space propagation, an additional 30-35 dB at small distances is noticed. A simplified temperature increase prediction scheme based on SAR is presented in [50]. It is argued that considering energy consumption is not enough and that the tissue is sensitive to temperature increase. The influence of a patient's body shape and position on the radiation pattern from an implanted radio transmitter has been studied in [51]. It is concluded that the difference between body shapes (i.e. male, female and child) are at least as large as the impact of a patient's arm movements.

\subsubsection{Along the Body}

Most of the devices used in a WBAN however are attached on the body. The propagation along the human body can be divided into line of sight (LOS) and nonline of sight (NLOS) situations. In the former, the curvature effects of the body are not taken into account as simulations are performed on a flat phantom or experiments are done at one side of the body. In the latter, the effect of propagation from the front of the body to the side or back are evaluated.

The channel model for line of sight (LOS) propagation along the human body was studied in [24,52-55], both by simulations and experiments. The studies were done for both narrowband and UWB signals. However, the results can be compared as the studies for UWB signals were performed in a band between 3 to $6 \mathrm{GHz}$ 
Table 2 Schematic overview of differences between Wireless Sensor Networks and Wireless Body Area Networks, based on [45].

\begin{tabular}{|c|c|c|}
\hline Challenges & Wireless Sensor Network & Wireless Body Area Network \\
\hline Scale & Monitored environment (meters / kilometers) & "Human body (centimeters / meters) \\
\hline Node Number & Many redundant nodes for wide area coverage & Fewer, limited in space \\
\hline Result accuracy & Through node redundancy & Through node accuracy and robustness \\
\hline Node Tasks & Node performs a dedicated task & Node performs multiple tasks \\
\hline Node Size & Small is preferred, but not important & Small is essential \\
\hline Network Topology & Very likely to be fixed or static & More variable due to body movement \\
\hline Data Rates & Most often homogeneous & Most often heterogeneous \\
\hline Node Replacement & Performed easily, nodes even disposable & Replacement of implanted nodes difficult \\
\hline Node Lifetime & Several years / months & $\begin{array}{l}\text { Several years / months, smaller battery capac- } \\
\text { ity }\end{array}$ \\
\hline Power Supply & $\begin{array}{l}\text { Accessible and likely to be replaced more easily } \\
\text { and frequently }\end{array}$ & $\begin{array}{l}\text { Inaccessible and difficult to replaced in an im- } \\
\text { plantable setting }\end{array}$ \\
\hline Power Demand & Likely to be large, energy supply easier & Likely to be lower, energy supply more difficult \\
\hline Energy Scavenging Source & Most likely solar and wind power & $\begin{array}{l}\text { Most likely motion (vibration) and thermal } \\
\text { (body heat) }\end{array}$ \\
\hline Biocompatibility & Not a consideration in most applications & A must for implants and some external sensors \\
\hline Security Level & Lower & Higher, to protect patient information \\
\hline Impact of Data Loss & Likely to be compensated by redundant nodes & $\begin{array}{l}\text { More significant, may require additional mea- } \\
\text { sures to ensure QoS and real-time data delivery. }\end{array}$ \\
\hline Wireless Technology & Bluetooth, ZigBee, GPRS, WLAN, ... & Low power technology required \\
\hline
\end{tabular}

and the narrowband system around $2.4 \mathrm{GHz}$ is relatively close to the band of $3 \mathrm{GHz}$. It was found that the path loss exponent $\eta$ is between 3 and 4, depending on the position of the device, e.g. the path loss on the arm is lower than the one on the trunk. It is claimed that this is probably due to the higher absorption in the larger volume of the trunk, and because the surface of the trunk is less flat than the surface of the stretched arm. The study in [53] shows a significant impact of the antenna height on the path loss. The closer the antenna is to the body, the higher the path loss: a difference of more than $20 \mathrm{~dB}$ is found for an antenna placed at 5 $\mathrm{mm}$ and $5 \mathrm{~cm}$. As the sensors and antennas of a Wireless Body Area Network will be designed to be as small as possible, the antenna will be close to the body which will result in a higher path loss.

In non-line of sight (NLOS) situations, there is no direct view between the sender and receiver. The EMwaves are diffracting around the body rather than having a direct path through the body. In $[16,54,55]$, a path loss exponent ranging from 5 to 6 was found. Thus a higher path loss along the NLOS channel than along the LOS channel was observed, due to diffraction around the human body ${ }^{2}$ and absorption of a larger amount of radiation by the body. In [56] the dominant propagation mechanism for the the ear-to-ear link, which can be regarded as a worst case scenario at the head due to the missing line-of-sight component, was identified. It was shown that for transmission from one side of the head to the opposite direct transmission can be

\footnotetext{
2 This is also referred to as creeping waves. A creeping wave is defined as the wave that is diffracted around the shadowed surface of a smooth body such as a sphere.
}

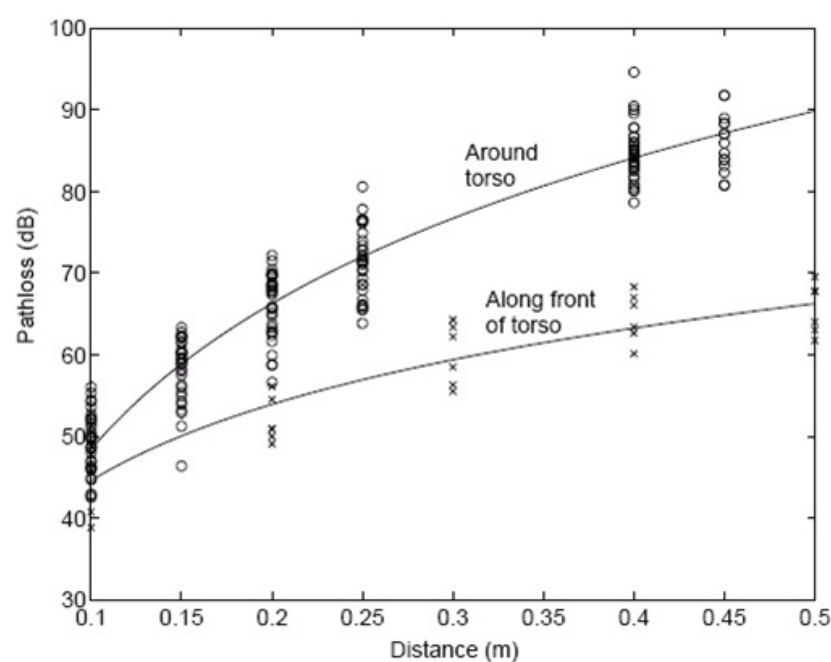

Fig. 5 Measured path loss versus the distance around (NLOS) and along the torso (LOS). It can be clearly seen that line-of sight communication experiences a lower path loss [58].

neglected due to reflections and the strong attenuation of the head.

The results above show that it is not always possible to assume single-hop communication along the body. Figure 5 shows the measured path loss for a LOS and NLOS scenario. Further, it is shown that in terms of energy efficiency, the use of multi-hop communication in a WBAN could lead to a more optimal network topology $[24,57]$. 


\subsection{Movement of the Body}

The movement of the body plays an important role in the strength of the received signal. In [58] it is shown that arm motions to the front and side of the body can have a small impact on the received power. More significant variations are found when the arms are moved so that they block the line of sight between the two antennas. In [59] a preliminary system model for gait analysis has been proposed. It is concluded that significant attenuation can occur (up to $20 \mathrm{~dB}$ ) when a body limb is moved in between the Tx and Rx antenna. According to [60] the movement of the limbs can induce an attenuation of $30 \mathrm{~dB}$ or more. A similar conclusion was found in an actual implementation [37] where the sensors communicate directly with the personal device using an RF-radio operating at $868 \mathrm{MHz}$. Loss rates of more than $50 \%$ where found when the body was in motion.

\subsection{Non-RF Communication}

Next to the propagation of radio waves, several researchers have investigated the possibility to transfer electronic data by capacitive and galvanic coupling, also called body-coupled communication (BCC). These radios work at low frequencies (ranging from $10 \mathrm{kHz}$ to $10 \mathrm{MHz}$ ). Zimmerman [61] first showed the potential of interference-free ultra low power data communication through the human body. High variations of the transmission attenuation have been observed at different locations of the body. Galvanic coupling promises to be a potential communication technology for sensor application on the thorax and for short distances on the limbs [62]. This technology can also be used to exchange data from one body to another by for example shaking hands [63]. In [64] OsteoConduct is presented, where the human musculoskeletal system is used to transmit data and information in a low-power, secure, nonintrusive fashion. Although this research looks promising, only very low data rates can be achieved ( $5 \mathrm{bits} / \mathrm{s}$ ).

The idea of BCC is further exploited by [65] for bootstrapping WBANs. They argue to equip the nodes with both RF and BCC capabilities. As a BCC is restricted to a person's body, the BCC can be used to discover and identify sensor nodes on the same body and for waking up RF radios from low-power sleep mode.

\section{MAC layer}

The number of MAC-protocols specifically developed for WBANs is limited. As networking in Wireless Sen- sor Networks has some points in common with networking in WBANs, it is useful to consider the research in MAC-protocols designed for WSNs. An overview can be found in $[66,67]$. Two major categories are contentionbased and schedule-based. For the former, CSMA/CA is a typical example, while TDMA is a typical scheme for the latter. The advantages of contention-based approaches are the simplicity, its infrastructure-free ad hoc feature and good adaptability to traffic fluctuation, especially for low load. Schedule-based approaches on the other hand are free of idle listening, overhearing and packet collisions because of the lack of medium competition, but require tight time synchronization. The most commonly used technique for reducing energy consumption in contention-based protocols is controlling the power and duty cycle of the radio.

Some implementations of WBANs use Bluetooth (IEEE 802.15.1) [68]. This was developed as a cable replacement and does not support (or only very limited) multi-hop communication. It has a complex protocol stack and a high energy consumption compared to IEEE 802.15.4. It is therefore not suited to be used in a WBAN.

Most current implementations of WBANs use IEEE 802.15.4 [69] or ZigBee [70] as enabling technology. As most of the radios used in a WBAN are based on an IEEE 802.15.4 compliant chip set, some researchers have adapted the IEEE 802.15.4 MAC-protocol to make it more suitable for WBANs. We will therefore first discuss the usefulness of IEEE 802.15.4 for medical networking. In a second part, other MAC-protocols for WBANs will discussed. An overview is given in Table 3. It can be noticed that all proposed MAC-protocols use slotted communication and assume a star topology by using master-slave communication. However, in the previous section it has been shown that single-hop communication is not always possible.

\subsection{IEEE 802.15.4}

In [71] the star network configuration of the IEEE 802.15.4 standard at $2.4 \mathrm{GHz}$ was considered for a WBAN. The analysis considers quite extensively a very low data rate star network with 10 body implanted sensors transmitting data 1 to 40 times per hour. The analysis focuses on the effect of crystal tolerance, frame size and the usage of IEEE 802.15.4 Guaranteed Time Slots (GTS) on a node lifetime. The main consideration in this work was the long-term power consumption of devices. The results show that IEEE 802.15.4 provides a limited answer for medical sensor networking when configured in non-beacon mode with low data rate asymmetric traf- 
Table 3 Schematic overview of MAC protocols in a WBAN.

\begin{tabular}{|l|c|c|c|c|c|}
\hline MAC-protocol & $\begin{array}{c}\text { IEEE 802.15.4 } \\
\text { based }\end{array}$ & TDMA based & CSMA based & $\begin{array}{c}\text { Star topology } \\
\text { (master/slave) }\end{array}$ & $\begin{array}{c}\text { Time Synchronization available } \\
\text { in the protocol }\end{array}$ \\
\hline \hline Timmons [71] & $\sqrt{ }$ & $\sqrt{ }$ & $\sqrt{ }$ & $\sqrt{ }$ \\
BSN-MAC [72] & $\sqrt{ }$ & $\sqrt{\mid 2} \operatorname{mixed}$ & $\sqrt{ }$ & \\
Lamprinos [73] & & $\sqrt{ }$ & & $\sqrt{ }$ \\
Omeni [74] & & $\sqrt{ }$ & & $\sqrt{ }$ & \\
H-MAC [75] & & & \\
\hline
\end{tabular}

fic. Beacon mode can also be used, but with even more severe restrictions on data rate and crystal tolerance.

Another adaptation is BSN-MAC [72]. The coordinator controls the communication by varying the superframe structure of IEEE 802.15.4. This divides the time axis in a contention-free and contention-based period. The sensors provide real-time feedback to a BSN coordinator with application-specific and sensor-specific information. Hence, based on the feedback the BSN coordinator can make dynamic adjustments for the length of the contention-free and contention-based period to achieve better performance in energy efficiency and latency.

Both [76] and [77] come to the conclusion that although 802.15.4 can provide QoS, the technology is not scalable in terms of power consumption and can not be used as a single solution for all WBAN applications.

It can be concluded that IEEE 802.15.4 is not the best solution for supporting communication in WBANs. Although it can be used for a quick (and easy) implementation, the results are rather poor. IEEE 802.15.4 was not designed to support WBANs. Specialized MAC protocols are needed.

\subsection{WBAN Specific Protocols}

One of the few MAC-protocols for WBANs was proposed by Lamprinos et al. [73]. They use a master-slave architecture and, to avoid idle listening, all slaves are locked in the Rx-slot of the master and go in standby at the same time. The main drawback of this protocol is that some slaves will have a low duty cycle whereas the nodes that are serviced later have a higher duty cycle. The protocol was implemented nor simulated. An adaptation of this protocol was used in [78]. This protocol divides time into frames in which only one node is allowed to transmit. The scheduling order is derived by applying the Earliest Deadline First algorithm. Omeni et al. [74] propose a MAC protocol for a star-networked WBAN that supports TDMA to reduce the probability of collision and idle listening. Each slave node is assigned a slot by the central node. When an alarm occurs at one of the nodes, the node can be assigned an extra slot for direct communication. The protocol has been evaluated on a Sensium platform. The H-MAC protocol [75] uses the human heartbeat rhythm information to perform time synchronization for TDMA. The biosensors can thus achieve time synchronization without having to turn on their radio. The algorithm is verified with real world data but assumes a certain buffer. The simulations do not show the energy gain and the protocol is designed for a star-topology WBAN only.

\subsection{IEEE 802.15.6}

Started as a Study Group in 2006 and motivated by the increasing research and industry interest in WBANs, the IEEE Standards Association decided to form the IEEE 802.15 Task Group 6 in November 2007. It describes itself as follows: The IEEE 802.15 Task Group $6(B A N)$ is developing a communication standard optimized for low power devices and operation on, in or around the human body (but not limited to humans) to serve a variety of applications including medical, consumer electronics / personal entertainment and other [13].

Project Authorization Request (PAR) 07-0575 presents an extended description of the task group [79]. It stresses the fact that current WPANs do not meet medical communication guidelines, because of the proximity to human tissue. Moreover, WPAN technology is said not to support Quality of Service, low power operation and noninterference, all required to support WBAN applications. Based on the responses to the Call for Applications [80], the PAR also outlines a large number of applications that can be served by the proposed standard, going from classical medical usage, e.g. EEG and ECG monitoring, to personal entertainment systems.

In 2008, a Call for Proposals on physical layer and MAC layer protocols was issued [81]. The large number of responses, 64 in total, confirmed the industry interest. Currently, the responses are being evaluated at monthly meetings, while some proposals are merged. 
The creation of the IEEE 802.15 Task Group 6 and the work on an IEEE 802.15.6 standard stresses the importance of the research with respect to WBANs.

\section{Network layer}

Developing efficient routing protocols in WBANs is a nontrivial task because of the specific characteristics of the wireless environment. First of all, the available bandwidth is limited, shared and can vary due to fading, noise and interference, so the protocol's amount of network control information should be limited. Secondly, the nodes that form the network can be very heterogeneous in terms of available energy or computing power.

Although a lot of research is being done toward energy efficient routing in ad hoc networks and WSNs [82], the proposed solutions are inadequate for WBANs. For example, in WSNs maximal throughput and minimal routing overhead are considered to be more important than minimal energy consumption. Energy efficient adhoc network protocols only attempt to find routes in the network that minimize energy consumption in terminals with small energy resources, thereby neglecting parameters such as the amount of operations (measurements, data processing, access to memory) and energy required to transmit and receive a useful bit over the wireless link. Most protocols for WSNs only consider networks with homogeneous sensors and a many-to-one communication paradigm. In many cases the network is considered as a static one. In contrast, a WBAN has heterogeneous mobile devices with stringent real-time requirements due to the sensor-actuator communication. Specialized protocols for WBANs are therefore needed.

In the following, an overview of existing routing strategies for WBANs is given. They can be subdivided in two categories: routing based on the temperature of the body and cluster based protocols.

\subsection{Temperature Routing}

When considering wireless transmission around and on the body, important issues are radiation absorption and heating effects on the human body. To reduce tissue heating the radio's transmission power can be limited or traffic control algorithms can be used. In [83] rate control is used to reduce the bioeffects in a single-hop network. Another possibility is a protocol that balances the communication over the sensor nodes. An example is the Thermal Aware Routing Algorithm (TARA) that routes data away from high temperature areas

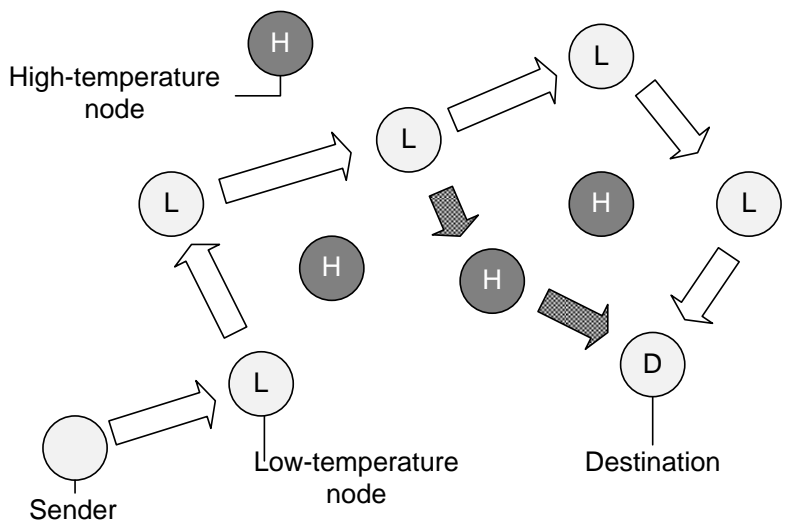

Fig. 6 An example of LTR and ALTR. The white arrows indicate the LTR-path. The shaded arrows show the adapted path of ALTR. When the path has three hops, the routing algorithm switches to shortest path routing.

(hot spots) [50]. Packets are withdrawn from heated zones and rerouted through alternate paths. TARA suffers from low network lifetime, a high ratio of dropped packets and does not take reliability into account. An improvement of TARA is Least Temperature Routing (LTR) and Adaptive Least Temperature Routing (ALTR) [84] that reduces unnecessary hops and loops by maintaining a list in the packet with the recently visited nodes. ALTR switches to shortest hop routing when a predetermined number of hops is reached in order to lower the energy consumption. An example of LTR and ALTR is given in Fig. 6. A smarter combination of LTR and shortest path routing is Least Total Route Temperature (LTRT) [36]. The node temperatures are converted into graph weights and minimum temperature routes are obtained. A better energy efficiency and a lower temperature rise is obtained, but the protocol has as main disadvantage that a node needs to know the temperature of all nodes in the network. The overhead of obtaining this data was not investigated.

\subsection{Cluster Based Routing}

"Anybody" [35] is a data gathering protocol that uses clustering to reduce the number of direct transmissions to the remote base station. It is based on LEACH [85] that randomly selects a cluster head at regular time intervals in order to spread the energy dissipation. The cluster head aggregates all data and sends it to the base station. LEACH assumes that all nodes are within sending range of the base station. Anybody solves this problem by changing the cluster head selection and constructing a backbone network of the cluster heads. The energy efficiency is not thoroughly investigated and reliability is not considered. Another improvement of 
LEACH is Hybrid Indirect Transmissions (HIT) [86], which combines clustering with forming chains. Doing so, the energy efficiency is improved. Reliability, however, is not considered.

This overview clearly shows that routing protocols for WBANs is an emerging area of research, the protocols described above were only developed in the last two years.

\section{Cross-layer Protocols}

Cross-layer design is a way to improve the efficiency of and interaction between the protocols in a wireless network by combining two or more layers from the protocol stack. This research has gained a lot of interest in sensor networks $[87,88]$. However, little research has been done for WBANs.

Ruzelli et al. propose a cross-layer energy efficient multi-hop protocol built on IEEE 802.15.4 [46]. The network is divided into time zones where each one takes turn in the transmission. The nodes in the farthest timezone start the transmission. In the next slot, the farthest but one sends its data and so on until the sink is reached. The protocol almost doubles the lifetime compared to regular IEEE 802.15.4. The protocol was developed for regular sensor networks, but the authors claim its usefulness for WBANs.

CICADA [34] uses a data gathering tree and controls the communication using distributed slot assignment. It has low packet loss and high sleep ratios while the network flexibility is preserved. It also enables twoway communication. Data-aggregation and the use of a duty cycle even further improved the lifetime of the network.

Another approach for cross layering is completely discarding the layered structure and implementing the required functionality in different modules which interact and can be changed easily [89]. A first attempt for WBANs using this method is described in [90].

\section{Quality of Service}

The research on QoS solutions is extensive for general ad hoc networks. However, these QoS solutions are designed for more powerful devices which are often line-powered. Most of these solutions do not apply to WSN or WBAN applications. Several QoS solutions specific for WSNs have been proposed, but these solutions mainly focus on one or a few QoS features such as reliability, delay, bandwidth specification or reservation [91]. For WBANs, researchers have shown little effort to provide QoS solutions.
In [92] the reliability of CICADA was evaluated and additional mechanisms were proposed in order to improve the reliability even further, such as the randomization of schemes and overhearing the control messages sent by the siblings.

BodyQos [93] addresses three unique challenges brought by BSN applications. It uses an asymmetric architecture where most of the processing is done at the central device. Second, they have developed a virtual MAC (V-MAC) that can support a wide variety of different MACs. Third, an adaptive resource scheduling strategy is used in order to make it possible to provide statistical bandwidth guarantees as well as reliable data communication in WBANs. The protocol has been implemented in nesC on top of TinyOS.

The desired quality of service will affect the energy consumption. For example, to obtain a lower packet loss, the transmit power can be increased, which raises the energy consumption. It is therefore important to achieve the right balance between power consumption and the desired reliability of the system.

\section{Security}

The communication of health related information between sensors in a WBAN is subject to the following security requirements: data confidentiality, data authenticity, data integrity and data freshness [94]. Data confidentiality means that the transmitted information is strictly private and can only be accessed by authorized persons, e.g. the doctor attending the patient. It is usually achieved by encrypting the information before sending it using a secret key and can be both symmetrically and asymmetrically. Data authenticity provides a means for making sure that the information is sent by the claimed sender. For this, a Message Authentication Code $\left(M A C^{3}\right)$ is calculated using a shared secret key. Data integrity makes sure that the received information has not been tampered with. This can be inspected by verifying the $M A C$. Data freshness guarantees that the received data is recent and not a replayed old message to cause disruption. A much used technique is to add a counter which is increased every time a message is sent.

The security mechanisms employed in WSNs do generally not offer the best solutions to be used in WBANs for the latter have specific features that should be taken into account when designing the security architecture. The number of sensors on the human body, and the range between the different nodes, is typically quite limited. Furthermore, the sensors deployed in a WBAN

$3 M A C$ is written in italic in order to avoid confusion with the abbreviation of Medium Access Control 
are under surveillance of the person carrying these devices. This means that it is difficult for an attacker to physically access the nodes without this being detected. When designing security protocols for WBANs, these characteristics should be taken into account in order to define optimized solutions with respect to the available resources in this specific environment. Although providing adequate security is a crucial factor in the acceptance of WBANs, little research has been done in this specific field. One of the most crucial components to support the security architecture is its key management. Further, security and privacy protection mechanisms use a significant part of the available resources and should therefore be energy efficient and lightweight.

A solution for data integrity and freshness was proposed in [95]. Their integrity algorithm is based on the measurement of a permissible round trip time threshold and is computational feasible. Authentication is done by calculating a $M A C$ with a random sequence of numbers. This sequence is determined at the initialization phase.

In [96] a security mechanism was added to CICADA. Doing so, CICADA-S became one of the first protocols where appropriate security mechanisms are incorporated into the communication protocol while addressing the life-cycle of the sensors. It was shown that the integration of key management and secure, privacy preserving communication techniques has low impact on the power consumption and throughput.

Another promising solution for key management is the use of biometrics. Biometrics is a technique commonly known as the automatic identification and verification of an individual by his or her physiological and/or behavioral characteristics [97]. In [12] an algorithm based on biometric data is described that can be employed to ensure the authenticity, confidentiality and integrity of the data transmission between the personal device and all other nodes. Algorithms that use the heartbeat to generate a key are proposed in $[98,99]$.

In [65] body-coupled communication (BCC) is used to associate new sensors in a WBAN. As BCC is limited to the body, this techniques can be used to authenticate new sensors on the body.

The developers of WBANs will have to take into account the privacy issues. After all, a WBAN can be considered as a potential threat to freedom, if the applications go beyond "secure" medical usage, leading to a Big Brother society. Social acceptance would be the key to this technology finding a wider application. Therefore, considerable effort should be put in securing the communication and making sure that only authorized persons can access the data.

\section{Existing Projects}

Several research groups and commercial vendors are already developing the first prototypes of WBANs. However, this research mainly focuses on building a system architecture and service platform and in lesser extent on developing networking protocols. In this section, we provide a non-exhaustive overview of projects for WBANs.

Otto et al. [6] and Jovanov et al. [32] present a system architecture which both handles the communication within the WBAN and between the WBANs and a medical server in a multi-tier telemedicine system. The communication between the sensors and the sink is single-hop, slotted and uses ZigBee or Bluetooth. The slots are synchronized using beacons periodically sent by the sink. They use off-the-shelf wireless sensors to design a prototype WBAN such as the Tmote sky platform from formerly Moteiv [100], now sentilla [101].

The Tmote sky platform is also used in the CodeBlueproject $[102,103]$ where WBANs are used in rapid disaster response scenarios. A wearable computer attached to the patient's wrist, i.e. a Tmote Sky mote, forms an ad hoc wireless network with a portable tablet PC. They developed a wireless two-lead ECG, a wireless pulse oximeter sensor and a wireless electromyogram (EMG).

Ayushman [104] is a sensor network based medical monitoring infrastructure that can collect, query and analyze patient health information in real-time. A wireless ECG, gait monitoring and environment monitoring was developed using off-the-shelf components with a Mica2 wireless transceiver. Further, the necessary software for consulting the data at a remote client was developed.

The Human++ project by IMEC-NL [10] aims "to achieve highly miniaturized and autonomous sensor systems that enable people to carry their personal body area network.". An ambulatory EEG/ECG system with a transmitter working on $2.4 \mathrm{GHz}$ was developed. This system can run for approximately 3 months using 2 AA batteries. In order to obtain a longer autonomy, the project also investigates energy scavenging with thermoelectric generators (TEG). In 2006, a wireless pulse oximeter was presented, fully powered by the patient's body heat. Further, the project investigates new wireless technologies such as UWB to make an ultra-low power transmitter.

The European MobiHealth project [105] provides a complete end-to-end mHealth platform for ambulant patient monitoring, deployed over UMTS and GPRS networks. The MobiHealth patient/user is equipped with different sensors that constantly monitor vital signals, 
e.g. blood pressure, heart rate and electrocardiogram (ECG). Communication between the sensors and the personal device is Bluetooth or ZigBee based and is single-hop. The major issues considered are security, reliability of communication resources and QoS guarantees.

The French project BANET [106] aims to provide a framework, models and technologies to design optimized wireless communication systems targeting the widest range of WBAN-based applications, in the consumer electronics, medical and sport domains. They focus on the study of the WBAN propagation channel, $\mathrm{MAC}$ protocols and coexistence of WBANs and other wireless networks.

The German BASUMA-project (Body Area System for Ubiquitous Multimedia Applications) [107] aims at developing a full platform for WBANs. As communication technique, a UWB-frontend is used and a MACprotocol based on IEEE 802.15.3. This protocol also uses time frames divided into contention free periods (with time slots) and contention access periods (CSMA/CA

A flexible and efficient WBASN solution suitable for a wide range of applications is developed in [108]. The focus lies on posture and activity recognition applications by means of practical implementation and on-thefield testing. The sensors are WiMoCA-nodes, where sensors are represented by tri-axial integrated MEMS accelerometers.

The Flemish IBBT IM3-project (Interactive Mobile Medical Monitoring) focuses on the research and implementation of a wearable system for health monitoring [109]. Patient data is collected using a WBAN and analyzed at the medical hub worn by the patient. If an event (e.g. heart rhythm problems) is detected, a signal is sent to a health care practitioner who can view and analyze the patient data remotely.

\section{Open Research Issues}

The discussions above clearly show that, although a lot of research is going on, still a lot of open issues exist.

Several researchers have already started studying the propagation of electromagnetic waves in and on the body and a few models for the physical layer are proposed. It should be noticed that none of them take the movements of the body into account, although movements can have severe impact on the received signal strength, as described in Section 5.2. Further, new emerging technologies such as galvanic coupling and transformation of information via the bones offer promising results and need to be investigated more thoroughly.

Although some protocols already exist that take care of the data link layer and networking, this area still has a lot of open research issues. On the data link layer, more WBAN specific MAC-protocols need to be developed that take into account the movement of the body, i.e. the mobility of the nodes, additional low-power features such as an adaptive duty cycle for lowering the idle listening and overhearing, the use of the human physiology such as heart beat to ensure time synchronization and so on. Concerning the network layer, a promising research track is the combination of thermal routing with more energy efficient mechanisms. More efficient QoS-mechanisms are needed, for example based on the BodyQos framework. Other interesting open research issues are mobility support embedded in the protocol, security, inter operability and so on. In order to define a globally optimal system, it might be necessary to unite several of these mechanisms in a cross-layer protocol.

The use energy scavenging was not addressed in detail in this paper but is nevertheless important. With a smart combination of lower energy protocols and energy scavenging, the optimal solution for achieving auAtonomous Body Area Networks can be reached. For a WBAN, energy scavenging from on-body sources such as body heat and body vibration seems very well suited. The ultimate goal is to create a small and smart bandaid containing all necessary technology for sensing and communication with a base station. Very preliminary examples can be found in the Sensium-platform [74] and the Human++-project [10].

\section{Conclusions}

In this survey, we have reviewed the current research on Wireless Body Area Networks. In particular, this work presents an overview of the research on the propagation in and on the human body, MAC-protocols, routing protocols, Quality of Service and security. To conclude, a list of research projects is given and open research issues are discussed.

A WBAN is expected to be a very useful technology with potential to offer a wide range of benefits to patients, medical personnel and society through continuous monitoring and early detection of possible problems. With the current technological evolution, sensors and radios will soon be applied as skin patches. Doing so, the sensors will seamlessly be integrated in a WBAN. Step by step, these evolutions will bring us closer to a fully operational WBAN that acts as an enabler for improving the Quality of Life. We feel that this review can be considered as a source of inspiration for future research directions. 


\section{References}

1. D. Cypher, N. Chevrollier, N. Montavont, and N. Golmie, "Prevailing over wires in healthcare environments: benefits and challenges," IEEE Communications Magazine, vol. 44, no. 4, pp. 56-63, Apr. 2006.

2. R. S. H. Istepanian, E. Jovanov, and Y. T. Zhang, "Guest editorial introduction to the special section on m-health: Beyond seamless mobility and global wireless health-care connectivity," Information Technology in Biomedicine, IEEE Transactions on, vol. 8, no. 4, pp. 405-414, Dec. 2004.

3. K. Van Dam, S. Pitchers, and M. Barnard, "Body area networks: Towards a wearable future," in Proceedings of WWRF kick off meeting, Munich, Germany, 6-7 March 2001.

4. R. Schmidt, T. Norgall, J. Mörsdorf, J. Bernhard, and T. von der G"un, "Body area network ban-a key infrastructure element for patient-centered medical applications." Biomedizinische Technik. Biomedical engineering, vol. 47, no. 1, pp. 365-368, 2002.

5. B. Gyselinckx, C. Van Hoof, J. Ryckaert, R. F. Yazicioglu, P. Fiorini, and V. Leonov, "Human++: autonomous wireless sensors for body area networks," in Custom Integrated Circuits Conference, 2005. Proceedings of the IEEE 2005, Sep. 2005, pp. 13-19.

6. C. Otto, A. Milenkovic, C. Sanders, and E. Jovanov, "System architecture of a wireless body area sensor network for ubiquitous health monitoring," Journal of Mobile Multimedia, vol. 1, no. 4, pp. 307-326, 2006.

7. B. Lo and G.-Z. Yang, "Body sensor networks: Infrastructure for life science sensing research," in Life Science Systems and Applications Workshop, 2006. IEEE/NLM, Bethesda, MD, Jul. 2006, pp. 1-2.

8. A. D. Jurik and A. C. Weaver, "Remote medical monitoring," Computer, vol. 41, no. 4, pp. 96-99, 2008.

9. S. Park and S. Jayaraman, "Enhancing the quality of life through wearable technology," IEEE Engineering in Medicine and Biology Magazine, vol. 22, no. 3, pp. 41-48, May/Jun. 2003.

10. B. Gyselinckx, R. Vullers, C. V. Hoof, J. Ryckaert, R. F. Yazicioglu, P. Fiorini, and V. Leonov, "Human++: Emerging technology for body area networks," in Very Large Scale Integration, 2006 IFIP International Conference on, Oct. 2006, pp. $175-180$.

11. "IEEE standard for safety levels with respect to human exposure to radio frequency electromagnetic fields, $3 \mathrm{khz}$ to 300 ghz," 1999.

12. C. C. Y. Poon, Y.-T. Zhang, and S.-D. Bao, "A novel biometrics method to secure wireless body area sensor networks for telemedicine and m-health," IEEE Communications Magazine, vol. 44, no. 4, pp. 73-81, Apr. 2006.

13. IEEE 802.15 WPAN Task Group 6 Body Area Networks. [Online]. Available: http://www.ieee802.org/15/pub/SGmban.html

14. World Health Organization [online] http://www.who.int/mediacentre/factsheets/fs317/en/index.htm 30

15. International Diabetes Federation (IDF) [Online] http://www.idf.org/.

16. B. Latré, G. Vermeeren, I. Moerman, L. Martens, and P. Demeester, "Networking and propagation issues in body area networks," in 11th Symposium on Communications and Vehicular Technology in the Benelux, SCVT 2004, Ghent, Belgium, 9 November 2004.

17. E. Jovanov, D. Raskovic, A. O. Lords, P. Cox, R. Adhami, and F. Andrasik, "Synchronized physiological monitoring using a distributed wireless intelligent sensor system," in
Engineering in Medicine and Biology Society, 2003. Proceedings of the 25th Annual International Conference of the IEEE, vol. 2, Sep. 2003, pp. 1368-1371.

18. S. Drude, "Requirements and application scenarios for body area networks," in Mobile and Wireless Communications Summit, 200\%. 16th IST, Budapest, Hungary, Jul. 2007, pp. $1-5$.

19. E. Krames, "Implantable devices for pain control: spinal cord stimulation and intrathecal therapies," Best Practice E Research Clinical Anaesthesiology, vol. 16, no. 4, pp. 619 649, December 2002.

20. H.-B. Li, K.-i. Takizawa, B. Zhen, and R. Kohno, "Body area network and its standardization at IEEE 802.15.MBAN," in Mobile and Wireless Communications Summit, 200\%. 16th IST, Budapest, Hungary,, Jul. 2007, pp. $1-5$.

21. L. Theogarajan, J. Wyatt, J. Rizzo, B. Drohan, M. Markova, S. Kelly, G. Swider, M. Raj, D. Shire, M. Gingerich, J. Lowenstein, and B. Yomtov, "Minimally invasive retinal prosthesis," in Solid-State Circuits, 2006 IEEE International Conference Digest of Technical Papers, Feb. 2006, pp. 99-108.

22. R. Hoyt, J. Reifman, T. Coster, and M. Buller, "Combat medical informatics: present and future," in Proceedings of the AMIA 2002 annual symposium, San Antonio, TX, November 2002, pp. 335-339.

23. I. F. Akyildiz, W. Su, Y. Sankarasubramaniam, and E. Cayirci, "A survey on sensor networks," IEEE Communications Magazine, vol. 40, no. 8, pp. 102-114, Aug. 2002.

24. T. Zasowski, F. Althaus, M. Stager, A. Wittneben, and G. Troster, "UWB for noninvasive wireless body area networks: channel measurements and results," in Ultra Wideband Systems and Technologies, 2003 IEEE Conference on, Nov. 2003, pp. 285-289.

25. T. Penzel, B. Kemp, G. Klosch, A. Schlogl, J. Hasan, A. Varri, and I. Korhonen, "Acquisition of biomedical signals databases," IEEE Engineering in Medicine and Biology Magazine, vol. 20, no. 3, pp. 25-32, May/Jun. 2001.

26. S. Arnon, D. Bhastekar, D. Kedar, and A. Tauber, "A comparative study of wireless communication network configurations for medical applications," IEEE [see also IEEE Personal Communications] Wireless Communications, vol. 10, no. 1, pp. 56-61, Feb. 2003.

27. B. Gyselinckx, J. Penders, and R. Vullers, "Potential and challenges of body area networks for cardiac monitoring, issue 6, supplement 1 , , isce 32 nd annual conference, november-december 2007, pages s165-s168." Journal of Electrocardiolog, vol. 40, no. 6, pp. S165-S168, NovemberDecember 2006, iSCE 32nd Annual Conference.

28. J. A. Paradiso and T. Starner, "Energy scavenging for mobile and wireless electronics," IEEE Pervasive Computing, vol. 04, no. 1, pp. 18-27, 2005

29. T. von Buren, P. D. Mitcheson, T. C. Green, E. M. Yeatman, A. S. Holmes, and G. Troster, "Optimization of inertial micropower generators for human walking motion," IEEE Sensors Journal, vol. 6, no. 1, pp. 28-38, Feb. 2006.

. International Commission on Non-ionizing Radiation Protection (ICNIRP), "Guidelines for limiting exposure to time-varying electric, magnetic, and electromagnetic fields (up to 300 ghz)," Health Physics, vol. 74, no. 4, pp. 494-522, apr 1998 .

31. U. Varshney and S. Sneha, "Patient monitoring using ad hoc wireless networks: reliability and power management," IEEE Communications Magazine, vol. 44, no. 4, pp. 49-55, Apr. 2006.

32. E. Jovanov, A. Milenkovic, C. Otto, and P. C. de Groen, "A wireless body area network of intelligent motion sensors 
for computer assisted physical rehabilitation," Journal of NeuroEngineering and Rehabilitation, vol. 2, no. 1, pp. 1623, March 2005.

33. A. Bhargava and M. Zoltowski, "Sensors and wireless communication for medical care," in Database and Expert Systems Applications, 2003. Proceedings. 14th International Workshop on, Sep. 2003, pp. 956-960.

34. B. Latré, B.Braem, I.Moerman, C. Blondia, E. Reusens, W. Joseph, and P. Demeester, "A low-delay protocol for multihop wireless body area networks," in 4th Annual International Conference on Mobile and Ubiquitous Systems: Networking 85 Services, 2007, Workshop PerNets, Philadelphia, PA, USA, 6-10 August 2007, pp. 479-486.

35. T. Watteyne, S. Augé-Blum, M. Dohler, and D. Barthel, "Anybody: a self-organization protocol for body area networks," in Second International Conference on Body Area Networks (BodyNets), Florence, Italy, 11-13 June 2007.

36. D. Takahashi, Y. Xiao, F. Hu, J. Chen, and Y. Sun, "Temperature-aware routing for telemedicine applications in embedded biomedical sensor networks," EURASIP Journal on Wireless Communications and Networking, vol. 2008, no. Article ID 572636, 2008, 11 pages.

37. A. Ylisaukko-oja, E. Vildjiounaite, and J. Mantyjarvi, "Five-point acceleration sensing wireless body area network - design and practical experiences," iswc, vol. 00, pp. 184$185,2004$.

38. N. T. Dokovski, A. T. van Halteren, and I. A. Widya, "Banip: Enabling remote healthcare monitoring with body area networks," in FIDJI 2003 International Workshop on Scientific Engineering of Distributed Java Applications, Luxembourg, ser. Lecture notes in Computer Science, N. Guelfi, E. Astesiano, and G. Reggio, Eds., vol. 2952/2004. Heidelberg: Springer Verlag, 2004, pp. 62-72.

39. K. E. Wac, R. Bults, A. van Halteren, D. Konstantas, and V. F. Nicola, "Measurements-based performance evaluation of $3 \mathrm{~g}$ wireless networks supporting m-health services," in Multimedia Computing and Networking 2005. Edited by Chandra, Surendar; Venkatasubramanian, Nalini. Proceedings of the SPIE, Volume 5680, pp. 176-187 (2004)., S. Chandra and N. Venkatasubramanian, Eds., Dec. 2004, pp. $176-187$.

40. A. Milenkovic, C. Otto, and E. Jovanov, "Wireless sensor networks for personal health monitoring: Issues and an implementation," Computer Communications, Wireless Sensor Networks and Wired/Wireless Internet Communications, vol. 29, no. 13-14, pp. 2521-2533, August 2006.

41. O. O. Olugbara, M. O. Adigun, S. O. Ojo, and P. Mudali, "Utility grid computing and body area network as enabler for ubiquitous rural e-healthcare service provisioning," in e-Health Networking, Application and Services, 2007 9th International Conference on, Taipei, Taiwan,, Jun. 2007, pp. 202-207.

42. I. Chlamtac, M. Conti, and J. Liu, "Mobile ad hoc networking: imperatives and challenges." Ad Hoc Networks, vol. 1, no. 1 , pp. 13-64, 2003.

43. I. F. Akyildiz and I. H. Kasimoglu, "Wireless sensor and actor networks: research challenges," Ad Hoc Networks, vol. 2, no. 2, pp. 351-367, October 2004.

44. T. Zasowski, "A system concept for ultra wideband (UWB) body area networks," Ph.D. dissertation, PhD Thesis, ETH Zürich, No. 17259, 2007.

45. G.-Z. Yang, Ed., Body Sensor Networks. Springer-Verlag London Limited, 2006.

46. A. G. Ruzzelli, R. Jurdak, G. M. O'Hare, and P. V. D. Stok, "Energy-efficient multi-hop medical sensor networking," in HealthNet '07: Proceedings of the 1st ACM SIGMOBILE international workshop on Systems and networking support for healthcare and assisted living environments. New York, NY, USA: ACM, 2007, pp. 37-42.

47. R. C. Shah and M. Yarvis, "Characteristics of on-body 802.15.4 networks," in Wireless Mesh Networks, 2006. WiMesh 2006. 2nd IEEE Workshop on, Reston, VA, USA, 2006, pp. 138-139.

48. T. S. Rappaport, Wireless Communication: Principles and Practice 2nd edition. Prentice Hall, 2002.

49. S. K. S. Gupta, S. Lalwani, Y. Prakash, E. Elsharawy, and L. Schwiebert, "Towards a propagation model for wireless biomedical applications," in Communications, 2003. ICC '03. IEEE International Conference on, vol. 3, May 2003, pp. $1993-1997$.

50. Q. Tang, N. Tummala, S. K. S. Gupta, and L. Schwiebert, "Communication scheduling to minimize thermal effects of implanted biosensor networks in homogeneous tissue," IEEE Transactions on Biomedical Engineering, vol. 52, no. 7, pp. 1285-1294, Jul. 2005.

51. A. J. Johansson, "Wave-propagation from medical implants-influence of body shape on radiation pattern," in 24th Annual Conference and the Annual Fall Meeting of the Biomedical Engineering Society, Proceedings of the Second Joint EMBS/BMES Conference, vol. 2, 2002, pp. 1409-1410.

52. E. Reusens, W. Joseph, G. Vermeeren, L. Martens, B. Latré, B. Braem, C. Blondia, and I. Moerman, "Path-loss models for wireless communication channel along arm and torso: Measurements and simulations," in IEEE Antennas and Propagation Society International Symposium 2007, Honolulu, HI, USA, 9-15 June 2007, pp. 336-339.

53. L. Roelens, S. Van den Bulcke, W. Joseph, G. Vermeeren, and L. Martens, "Path loss model for wireless narrowband communication above flat phantom," Electronics Letters, vol. 42 , no. 1 , pp. 10-11, Jan. 2006.

54. T. Zasowski, G. Meyer, F. Althaus, and A. Wittneben, "Propagation effects in UWB body area networks," in UltraWideband, 2005. ICU 2005. 2005 IEEE International Conference on, Sep. 2005, pp. 16-21.

55. A. Fort, J. Ryckaert, C. Desset, P. De Doncker, P. Wambacq, and L. Van Biesen, "Ultra-wideband channel model for communication around the human body," IEEE Journal on Selected Areas in Communications, vol. 24, pp. 927-933, Apr. 2006.

56. T. Zasowski, G. Meyer, F. Althaus, and A. Wittneben, "UWB signal propagation at the human head," IEEE Transactions on Microwave Theory and Techniques, Apr. 2006.

57. B. Braem, B. Latré, I. Moerman, C. Blondia, E. Reusens, W. Joseph, L. Martens, and P. Demeester, "The need for cooperation and relaying in short-range high path loss sensor networks," in First International Conference on Sensor Technologies and Applications (SENSORCOMM 2007), Valencia, Spain, 14-20 October 2007, pp. 566-571.

58. A. Fort, C. Desset, J. Ryckaert, P. De Doncker, L. Van Biesen, and P. Wambacq, "Characterization of the ultra wideband body area propagation channel," in UltraWideband, 2005. ICU 2005. 2005 IEEE International Conference on, Sep. 2005.

59. M. Di Renzo, R. M. Buehrer, and J. Torres, "Pulse shape distortion and ranging accuracy in uwbbased body area networks for fullbody motion capture and gait analysis," in IEEE Globecom 2007, November 2007, pp. 3775 - 3780.

60. D. Neirynck, "Channel characterisation and physical layer analysis for body and personal area network development," Ph.D. dissertation, University of Bristol, UK, November 2006. 
61. T. Zimmerman, "Personal area networks: Nearfield intrabody communication," IBM Systems Journal, vol. 35, no. 3, pp. 609-617, 1996.

62. M. S. Wegmueller, A. Kuhn, J. Froehlich, M. Oberle, N. Felber, N. Kuster, and W. Fichtner, "An attempt to model the human body as a communication channel," IEEE Transactions on Biomedical Engineering, vol. 54, no. 10, pp. 18511857, Oct. 2007.

63. K. Hachisuka, Y. Terauchi, Y. Kishi, T. Hirota, K. Sasaki, H. Hosaka, and K. Ito, "Simplified circuit modeling and fabrication of intrabody communication devices," in SolidState Sensors, Actuators and Microsystems, 2005. Digest of Technical Papers. TRANSDUCERS '05. The 13th International Conference on, vol. 1, Jun. 2005, pp. 461-464.

64. L. Zhong, D. El-Daye, B. Kaufman, N. Tobaoda, T. Mohamed, and M. Liebschner, "Osteoconduct: Wireless bodyarea communication based on bone conduction," in Proc. Int. Conf. Body Area Networks (BodyNets), June 2007.

65. T. Falck, H. Baldus, J. Espina, and K. Klabunde, "Plug 'n play simplicity for wireless medical body sensors," Mob. Netw. Appl., vol. 12, no. 2-3, pp. 143-153, 2007.

66. I. Demirkol, C. Ersoy, and F. Alagoz, "MAC protocols for wireless sensor networks: a survey," IEEE Communications Magazine, vol. 44, no. 4, pp. 115-121, Apr. 2006.

67. P. Baronti, P. Pillai, V. Chook, S. Chessa, A.Gotta, and Y. F. Hu, "Wireless sensor networks: A survey on the state of the art and the 802.15.4 and zigbee standards," Computer Communications, vol. 30, no. 7, pp. 1665-1695, May 2007.

68. P. Johansson, M. Kazantzidis, R. Kapoor, and M. Gerla, "Bluetooth: an enabler for personal area networking," IEEE Network, vol. 15, no. 5, pp. 28-37, Sep./Oct. 2001.

69. IEEE 802.15.4-2003: IEEE Standard for Information Technology - Part 15.4: Wireless Medium Access Control and Physical Layer specifications for Low Rate Wireless Personal Area Networks.

70. ZigBee Alliance, official webpage: http://www.zigbee.org.

71. N. F. Timmons and W. G. Scanlon, "Analysis of the performance of IEEE 802.15.4 for medical sensor body area networking," in First Annual IEEE Communications Society Conference on Sensor and Ad Hoc Communications and Networks, 2004. IEEE SECON, Oct. 2004, pp. 16-24.

72. H. Li and J. Tan, "An ultra-low-power medium access control protocol for body sensor network," in 27th Annual International Conference of the Engineering in Medicine and Biology Society, 2005. IEEE-EMBS, Shanghai,, 2005, pp. 2451-2454.

73. I. E. Lamprinos, A. Prentza, E. Sakka, and D. Koutsouris, "Energy-efficient MAC protocol for patient personal area networks," in 27th Annual International Conference of the Engineering in Medicine and Biology Society, 2005. IEEEEMBS, Shanghai,, 2005, pp. 3799-3802.

74. O. C. Omeni, O. Eljamaly, and A. J. Burdett, "Energy efficient medium access protocol for wireless medical body area sensor networks," in Medical Devices and Biosensors, $200 \%$. ISSS-MDBS 200\%. 4th IEEE/EMBS International Summer School and Symposium on, Cambridge, UK, Aug. 2007, pp. 29-32.

75. H. Li and J. Tan, "Heartbeat driven medium access control for body sensor networks," in HealthNet '07: Proceedings of the 1st ACM SIGMOBILE international workshop on Systems and networking support for healthcare and assisted living environments. Puerto Rico, USA: ACM, 11 June 2007, pp. 25-30.

76. N. Golmie, D. Cypher, and O. Rebala, "Performance analysis of low rate wireless technologies for medical applications," Computer Communications, vol. 28, no. 10, pp. 1266-1275, June 2005.
77. D. Cavalcanti, R. Schmitt, and A. Soomro, "Performance analysis of 802.15 .4 and 802.11 e for body sensor network applications," in 4th International Workshop on Wearable and Implantable Body Sensor Networks (BSN 2007), vol. Volume 13. Springer Berlin Heidelberg, 2007, pp. 9-14.

78. E. Farella, A. Pieracci, L. Benini, and A. Acquaviva, "A wireless body area sensor network for posture detection," in ISCC '06: Proceedings of the 11th IEEE Symposium on Computers and Communications. Washington, DC, USA: IEEE Computer Society, 2006, pp. 454-459.

79. B. Heile, "IEEE 802.15 TG 6 PAR," IEEE15-07-0575/r9, IEEE-SA, December 2007.

80. D. Lewis, "802.15 TG 6 Call for Applications - Response Summary," IEEE15-08-0407r6, IEEE-SA, July 2008.

81. A. Astrin, "802.15 TG 6 Call for Proposals (CFP)," IEEE15-08-0829r1, IEEE-SA, November 2008.

82. K. Akkaya and M. Younis, "A survey on routing protocols for wireless sensor networks," Ad Hoc Networks, vol. 3, no. 3, pp. 325-349, 2005.

83. H. Ren and M. Q. H. Meng, "Rate control to reduce bioeffects in wireless biomedical sensor networks," in Mobile and Ubiquitous Systems - Workshops, 2006. 3rd Annual International Conference on, San Jose, CA,, Jul. 2006, pp. 1-7.

84. A. Bag and M. A. Bassiouni, "Energy efficient thermal aware routing algorithms for embedded biomedical sensor networks," in Mobile Adhoc and Sensor Systems (MASS), 2006 IEEE International Conference on, Vancouver, BC,, Oct. 2006, pp. 604-609.

85. W. R. Heinzelman, A. Chandrakasan, and H. Balakrishnan, "Energy-efficient communication protocol for wireless microsensor networks," in System Sciences, 2000. Proceedings of the 33rd Annual Hawaii International Conference on, Jan 2000, pp. 8020-8024.

86. M. Moh, B. J. Culpepper, L. Dung, T.-S. Moh, T. Hamada, and C.-F. Su, "On data gathering protocols for in-body biomedical sensor networks," in Global Telecommunications Conference, 2005. GLOBECOM '05. IEEE, vol. 5, Nov./Dec. 2005.

87. R. Madan, S. Cui, S. Lall, and N. A. Goldsmith, "Crosslayer design for lifetime maximization in interferencelimited wireless sensor networks," IEEE Transactions on Wireless Communications, vol. 5, no. 11, pp. 3142-3152, Nov. 2006.

88. T. Melodia, M. Vuran, and D. Pompil, "The state of the art in cross-layer design for wireless sensor networks," in EuroNGI Workshop on Wireless and Mobility, ser. LNCS 3883, July 2005, pp. 78-92.

89. E. De Poorter, B. Latré, I. Moerman, and P. Demeester, Sensor and Ad-Hoc Networks: Theoretical and Algorithmic Aspects, ser. Lecture Notes Electrical Engineering. Springer, June 2008, vol. 7, ch. Universal Framework for Sensor Networks.

90. B. Latré, E. De Poorter, I.Moerman, and P. Demeester, "Mofban: a lightweight framework for body area networks," Lecture Notes in Computer Science, proceedings of Embedded and Ubiquitous Computing (EUC 2007), vol. 4808, pp. 610-622, December 2007.

91. D. Chen and P. K. Varshney, "Qos support in wireless sensor networks: A survey," in Int. Conference on Wireless Networks (ICWN 2004). CSREA Press, June 2004.

92. B. Braem, B. Latré, C. Blondia, I. Moerman, and P. Demeester, "Improving reliability in multi-hop body sensor networks," in Second International Conference on Sensor Technologies and Applications (SENSORCOMM 2008), Cap Esterel, France, 25-31 August 2008, pp. 342-347.

93. G. Zhou, J. Lu, C.-Y. Wan, M. Yarvis, and J. Stankovic, "Bodyqos: Adaptive and radio-agnostic qos for body sensor networks," April 2008, pp. 565-573. 
94. S. Cherukuri, K. K. Venkatasubramanian, and S. K. S. Gupta, "Biosec: a biometric based approach for securing communication in wireless networks of biosensors implanted in the human body," in Parallel Processing Workshops, 2003. Proceedings. 2003 International Conference on, Oct. 2003, pp. 432-439.

95. V. B. Balasubramanyn, G. Thamilarasu, and R. Sridhar, "Security solution for data integrity inwireless biosensor networks," in Distributed Computing Systems Workshops, 2007. ICDCSW '0\%. 27th International Conference on, Toronto, Ont., Jun. 2007, pp. 79-79.

96. D. Singelée, B. Latré, B. Braem, M. De Soete, P. De Cleyn, B. Preneel, I. Moerman, and C. Blondia, "A secure crosslayer protocol for multi hop wireless body area networks," in 7th International Conference on AD-HOC Networks 8 Wireless (ADHOCNOW 2008), vol. LNCS 5198, France, 11-13 September 2008, pp. 94-107.

97. M. Guennoun, M. Zandi, and K. El-Khatib, "On the use of biometrics to secure wireless biosensor networks," in Information and Communication Technologies: From Theory to Applications, 2008. ICTTA 2008. 3rd International Conference on, Damascus, Apr. 2008, pp. 1-5.

98. S.-D. Bao, C. C. Y. Poon, Y.-T. Zhang, and L.-F. Shen, "Using the timing information of heartbeats as an entity identifier to secure body sensor network," Information Technology in Biomedicine, IEEE Transactions on, vol. 12, no. 6, pp. 772-779, Nov. 2008.

99. F. M. Bui and D. Hatzinakos, "Biometric methods for secure communications in body sensor networks: Resourceefficient key management and signal-level data scrambling," EURASIP Journal on Advances in Signal Processing, vol. 2008, 2008, article ID 529879, 16 pages.

100. Moteiv [online] http://www.moteiv.com.

101. sentilla [online] http://www.sentilla.com.

102. T. Gao, D. Greenspan, M. Welsh, R. R. Juang, and A. Alm, "Vital signs monitoring and patient tracking over a wireless network," in Engineering in Medicine and Biology Society, 2005. IEEE-EMBS 2005. 27th Annual International Conference of the, Shanghai,, 2005, pp. 102-105.

103. K. Lorincz, D. J. Malan, T. R. F. Fulford-Jones, A. Nawoj, A. Clavel, V. Shnayder, G. Mainland, M. Welsh, and S. Moulton, "Sensor networks for emergency response: Challenges and opportunities," IEEE Pervasive Computing, vol. 3, no. 4, pp. 16-23, 2004.

104. K. Venkatasubramanian, G. Deng, T. Mukherjee, J. Quintero, V. Annamalai, and S. Gupta, "Ayushman: A wireless sensor network based health monitoring infrastructure and testbed," in Distributed Computing in Sensor Systems, vol. Volume 3560/2005. Springer Berlin / Heidelberg, 2005, pp. 406-407.

105. A. T. van Halteren, R. G. A. Bults, K. E. Wac, D. Konstantas, I. A. Widya, N. T. Dokovski, G. T. Koprinkov, V. M. Jones, and R. Herzog, "Mobile patient monitoring: The mobihealth system," The Journal on Information Technology in Healthcare, vol. 2, no. 5, pp. 365-373, October 2004.

106. BANET project website [online] http://www.banet.fr.

107. T. Falck, J. Espina, J. P. Ebert, and D. Dietterle, "BASUMA - the sixth sense for chronically ill patients," in Wearable and Implantable Body Sensor Networks, 2006. $B S N$ 2006. International Workshop on, Cambridge, MA, USA, 3-5 April 2006, pp. 57-60.

108. E. Farella, A. Pieracci, L. Benini, L. Rocchi, and A. Acquaviva, "Interfacing human and computer with wireless body area sensor networks: the wimoca solution," Multimedia Tools and Applications, vol. 38, no. 3, pp. $337-363$, 2008.

109. IBBT IM3-project [online] http://projects.ibbt.be/im3. 